\title{
Expression of Toll-like receptors in the mucosa of patients with ulcerative colitis
}

\author{
YUJING FAN and BINGRONG LIU \\ Department of Gastroenterology, The Second Affiliated Hospital of Harbin Medical University, \\ Harbin, Heilongjiang 150080, P.R. China
}

Received April 11, 2014; Accepted December 1, 2014

DOI: $10.3892 /$ etm.2015.2258

\begin{abstract}
Patients with ulcerative colitis (UC) have a high risk of developing colorectal cancer. The aim of the present study was to evaluate the expression pattern of Toll-like receptors (TLRs) in the colonic mucosa of patients with UC. Colonic mucosal biopsy specimens were collected during colonoscopy from 30 patients with UC and 30 patients with normal findings as controls. The protein and mRNA expression levels of TLRs 1-4 and TLR9 were measured by immunohistochemistry and reverse transcription-quantitative polymerase chain reaction analysis, respectively. The results showed that the mRNA and protein expression of TLR2, TLR4 and TLR9, but not TLR1 and TLR3, was significantly increased in the colonic mucosa of patients with UC compared with that in the normal controls. TLR (TLR2, TLR4 and TLR9) immunoreactivity was found in the cytoplasm of epithelial cells in the mucosa, and occasionally in the endothelium of small vessels of the stromal tissues. In conclusion, TLR2, TLR4 and TLR9 expression may be important in the biological pathogenesis of UC. TLR alterations in the innate response system may contribute to the pathogenesis of UC.
\end{abstract}

\section{Introduction}

Ulcerative colitis (UC) is a relapsing and remitting disease characterized by acute non-infectious inflammation of the colorectal mucosa. The rectal mucosa is invariably affected. The incidence of UC is between 1.2 and 20.3 per 100,000 individuals per year, and its prevalence is between 7.6 and 246.0 per 100,000 individuals per year (1). Although the etiology of UC has yet to be fully elucidated, it has been suggested that environmental factors, such as gut microbiota, stimulate the inappropriate activation of mucosal immunity, thus leading

Correspondence to: Dr Bingrong Liu, Department of Gastroenterology, The Second Affiliated Hospital of Harbin Medical University, 246 Xuefu Road, Harbin, Heilongjiang 150080, P.R. China

E-mail: f_yu_jing@163.com

Key words: Toll-like receptor, ulcerative colitis, mucosa to inflammation (2). Intestinal homeostasis is dependent on a controlled innate immune response to the microbiota, which are recognized by Toll-like receptors (TLRs) on epithelial and immune cells (3).

TLRs comprise an important family of type I transmembrane receptors that allow immune cells to recognize pathogens and trigger inflammatory responses, and are expressed not only in a variety of immune cells but also in non-immune cells, such as fibroblasts and epithelial cells (4). TLRs are constitutively or inducibly expressed by a number of different cell types in the gastrointestinal tract, including intestinal epithelial cells and monocytes/macrophages, dendritic cells of the lamina propria and myofibroblasts, endothelial cells and adipocytes of the intestinal submucosa. When genetic predisposition or environmental stimuli impair mucosal or commensal homeostasis, certain intestinal diseases, such as Crohn's disease (CD), may develop (4-6).

Aberrant TLR signaling can induce tissue damage and barrier destruction through the overproduction of cytokines and chemokines and the loss of the commensal-mediated responses of colonic epithelial progenitors (7); however, although previous studies (8-10) have investigated the role of TLRs in inflammatory bowel disease (IBD), the expression of TLRs and their role in microbial recognition in innate immunity have not, to the best of our knowledge, been extensively evaluated in the colonic mucosa of patients with UC. The aim of the present study was to determine the alterations in the expression of the main conductors of the innate immune response, including TLR1, TLR2, TLR3, TLR4 and TLR9, in the colonic mucosa of patients with UC and normal controls. The protein and mRNA levels of TLRs 1-4 and TLR9 were evaluated by immunohistochemical techniques and reverse transcription-quantitative polymerase chain reaction analysis, respectively.

\section{Materials and methods}

Clinical samples. Following the provision of informed consent, unrelated patients with UC $(n=30)$ were recruited from the Outpatient Clinic at the Department of Gastroenterology of The Second Affiliated Hospital of Harbin Medical University (Harbin, China). The patients all fulfilled the diagnostic requirements of UC according to the Chinese Medical Association criteria. Unrelated healthy individuals with a 
Table I. Quantitative polymerase chain reaction primers used for the detection of TLRs.

\begin{tabular}{|c|c|c|}
\hline Gene & Primer sequence $\left(5^{\prime}-3^{\prime}\right)$ & Amplicon size (bp) \\
\hline TLR1 & & 102 \\
\hline Sense & AGGTCTTGCTGGTCTTAGGAGA & \\
\hline Antisense & TGTTTGTGGGGAACACAATGTG & \\
\hline TLR2 & & 160 \\
\hline Sense & TACTTTGTGGATGGTGTGGGTC & \\
\hline Antisense & GCTTTTTACAGCTTCTGTGAGC & \\
\hline TLR3 & & 196 \\
\hline Sense & AACTCAGAAGATTACCAGCCGC & \\
\hline Antisense & TCAGTCAAATTCGTGCAGAAGG & \\
\hline TLR4 & & 110 \\
\hline Sense & TCCATTTCAGCTCTGCCTTCAC & \\
\hline Antisense & ACACCACAACAATCACCTTTCG & \\
\hline TLR9 & & 154 \\
\hline Sense & CTGCGACCACGCTCCCAACCCC & \\
\hline Antisense & TCCCAGCCCACGGAACCAACTG & \\
\hline GAPDH & & 213 \\
\hline Sense & AAGAAGGTGGTGAAGCAGGC & \\
\hline Antisense & TCCACCACCCAGTTGCTGTA & \\
\hline
\end{tabular}

TLR, Toll-like receptor.

normal colonoscopy $(n=30)$ were randomly recruited from the same hospital. Two colonic biopsy specimens were collected from each participant for routine histological examination. One sample was fixed in $10 \%$ formalin for immunohistochemistry. The other sample was immediately frozen in liquid nitrogen and stored at $-80^{\circ} \mathrm{C}$ for later RNA extraction.

All subjects were of Chinese Han descent, and the patients and controls were matched for age and gender. This study was approved by the Ethical Review Committee of Research in Harbin Medical University.

Immunohistochemistry. Samples fixed in $10 \%$ formalin were subsequently embedded in paraffin, and sections of 4-mm thickness were cut from the formalin-fixed samples. The sectioned tissue was deparaffinized in xylene and then rehydrated in a graded ethyl alcohol series. For increased specificity and sensitivity, tissues were microwaved for $10 \mathrm{~min}$ for antigen retrieval. Following cooling and rinsing in distilled water, endogenous peroxide activity was blocked with $3 \% \mathrm{H}_{2} \mathrm{O}_{2}$ for $10 \mathrm{~min}$, and the samples were then rinsed in $0.01 \mathrm{~mol} / \mathrm{l}$ phosphate-buffered saline (PBS, $\mathrm{pH}$ 7.4) for $10 \mathrm{~min}$. The sections were subsequently preincubated with a protein blocking solution (ZSGB-BIO, Beijing, China) for $10 \mathrm{~min}$, prior to incubation with the primary polyclonal antibodies against TLR1 (cat. no. sc-30000; rabbit polyclonal), TLR2 (cat. no. sc-10739; rabbit polyclonal), TLR3 (cat. no. sc-32232; mose monoclonal), TLR4 (cat. no. sc-10741; rabbit polyclonal) and TLR9 (cat. no. sc-25468; rabbit polyclonal) (Santa Cruz Biotechnology, Inc., Santa Cruz, CA, USA) at dilutions of 1:300, 1:70, 1:300, 1:100 and 1:50, respectively, at $4^{\circ} \mathrm{C}$ overnight in a humid chamber. The slides were then washed three times in PBS and incubated with secondary biotinylated antibody (ZSGB-BIO) for $15 \mathrm{~min}$ at room temperature. The streptavidin-peroxidase method (ZSGB-BIO)was used to detect the antigen-antibody complexes, and diaminobenzidine (DAB) was used as the chromogen substrate. Peroxidase signals were visualized following $3 \mathrm{~min}$ of treatment with a DAB substrate-chromogen system (ZSGB-BIO). Finally, the sections were stained lightly with hematoxylin. For the negative control, PBS was used in place of the primary antibody. All sections were coded and independently examined by two investigators. The staining intensity was scored as negative or weak $(-)$, moderate $(+)$, strong $(++)$ or very strong $(+++)$.

Reverse transcription-quantitative polymerase chain reaction $(R T-q P C R)$ analysis. The expression rates of the TLR genes were analyzed through RT-qPCR analysis. Total RNA was extracted from fresh frozen tissue using TRIzol ${ }^{\mathrm{TM}}$ reagent (Invitrogen Life Technologies, Carlsbad, CA, USA) in accordance with the manufacturer's instructions. Total RNA $(1 \mu \mathrm{g})$ was subjected to reverse transcription using a Reverse Transcription system (Promega Biotech Co., Ltd., Beijing, China), according to the manufacturer's instructions. All primers (Sangon Biotech Co., Ltd., Shanghai, China) used in this study are shown in Table I. For the qPCR, $2 \mu \mathrm{l}$ cDNA, $1 \mu 1$ primers and $10 \mu \mathrm{l} \mathrm{qPCR}_{\mathrm{iQ}}{ }^{\mathrm{TM}} \mathrm{SYBR}^{\circledR}$ Green supermix (Bio-Rad, Hercules, CA, USA) was mixed to obtain a final volume of $20 \mu \mathrm{l}$. The reaction was followed by 40 cycles at $95^{\circ} \mathrm{C}$ for $30 \mathrm{sec}, 60^{\circ} \mathrm{C}$ for $30 \mathrm{sec}$ and $72^{\circ} \mathrm{C}$ for $30 \mathrm{sec}$. All reactions were performed in duplicate. Amplification of the expected single products was confirmed by dynamic melting curves and by electrophoresis on $1.5 \%$ agarose gels stained 

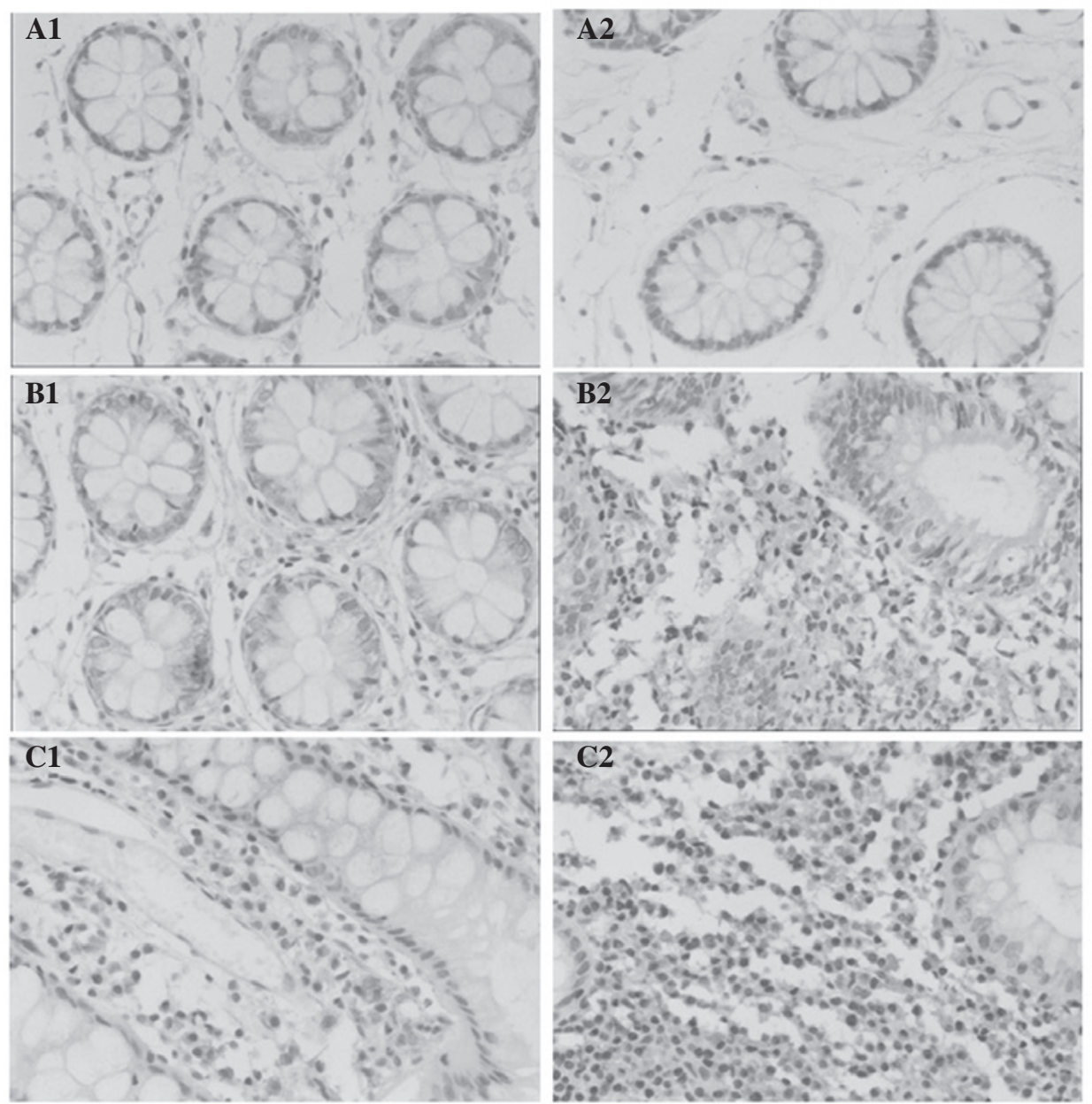

Figure 1. TLR protein immunoreactivity in the mucosa of (A1-C1) control subjects and (A2-C2) patients with UC: (A) TLR2, (B) TLR4 and (C) TLR9. Immunoreactivity was generally observed in the cytoplasm of epithelial cells and ocasionally in the endothelium of small vessels of the stromal tissues. Magnification, x400. TLR, Toll-like receptor; UC, ulcerative colitis.

with ethidium bromide. Fluorescence data were automatically collected and analyzed by iCycler iQ Optical Software (version 3.0a; Bio-Rad). The expression of TLRs 1-4 and TLR9 was examined and normalized to a constitutive gene (GAPDH), and relative induction was calculated using the $2^{(-\Delta \Delta C t)}$ method (11).

Statistical analysis. All data are presented as the mean \pm standard deviation and were analyzed using SPSS 16.0 statistical software (SPSS, Inc., Chicago, IL, USA). Statistical differences between groups were analyzed with non-parametric methods (Mann-Whitney U-test for unpaired data). The $\chi^{2}$ test was used to estimate mRNA values. $\mathrm{P}<0.05$ was considered to indicate a statistically significant difference.

\section{Results}

Expression of TLR proteins in mucosal biopsies. Immunohistochemical assessment for TLRs in the mucosal biopsies was performed using anti-TLR antibodies. Representative staining patterns for the TLRs are shown in Fig. 1. Positive staining for TLRs 1-4 and TLR9 were generally observed in the cytoplasm of epithelial cells, and TLR3 was also expressed on the cell membrane. In the stromal non-epithelial tissue, a weak positive reaction was additionally occasionally observed in the endothelium of the small vessels. The expression of TLR2, TLR4 and TLR9 was significantly stronger in the cytoplasm of epithelial cells from the patients with UC than those from the normal controls (Table II). The differences in the expression levels of TLR1 and TLR3 in the colonic mucosa between patients with UC and normal controls were not statistically significant (Table II).

Levels of TLR mRNA in mucosal biopsies of patients with UC and normal controls. Similar to the results for protein levels, the mRNA expression levels of TLR2, TLR4 and TLR9, but not TLR1 and TLR3, were significantly higher in the patients with UC than those in the normal controls (Fig. 2).

\section{Discussion}

UC comprises a group of chronic inflammatory disorders involving the mucosa of the colon. Despite a lack of extensive investigation into the role of the adaptive immune response in UC, evidence suggests an association between innate immunity and the pathology of the disease (12). The findings of numerous studies have indicated that the surface epithelium 
Table II. TLR protein detection by immunohistochemistry.

\begin{tabular}{|c|c|c|c|c|c|c|}
\hline \multirow[b]{2}{*}{ Protein } & \multicolumn{4}{|c|}{ Index of staining pattern } & \multirow[b]{2}{*}{ Mean rank } & \multirow[b]{2}{*}{ P-value } \\
\hline & Negative/weak (-) & Moderate (+) & Strong $(++)$ & Very strong $(+++)$ & & \\
\hline TLR1 & & & & & & 0.076 \\
\hline Control & 2 & 9 & 18 & 1 & 34.95 & \\
\hline $\mathrm{UC}$ & 0 & 19 & 11 & 0 & 26.95 & \\
\hline TLR2 & & & & & & 0.025 \\
\hline Control & 4 & 11 & 15 & 0 & 25.85 & \\
\hline $\mathrm{UC}$ & 2 & 9 & 16 & 3 & 35.15 & \\
\hline TLR3 & & & & & & 0.205 \\
\hline Control & 0 & 10 & 18 & 2 & 33.03 & \\
\hline $\mathrm{UC}$ & 2 & 12 & 15 & 1 & 27.97 & \\
\hline TLR4 & & & & & & 0.030 \\
\hline Control & 2 & 12 & 15 & 1 & 26.12 & \\
\hline $\mathrm{UC}$ & 0 & 9 & 19 & 2 & 34.88 & \\
\hline TLR9 & & & & & & 0.031 \\
\hline Control & 6 & 18 & 6 & 0 & 26.10 & \\
\hline $\mathrm{UC}$ & 3 & 13 & 14 & 0 & 34.90 & \\
\hline
\end{tabular}

$\mathrm{n}=30$ per group. TLR, Toll-like receptor; UC, ulcerative colitis.

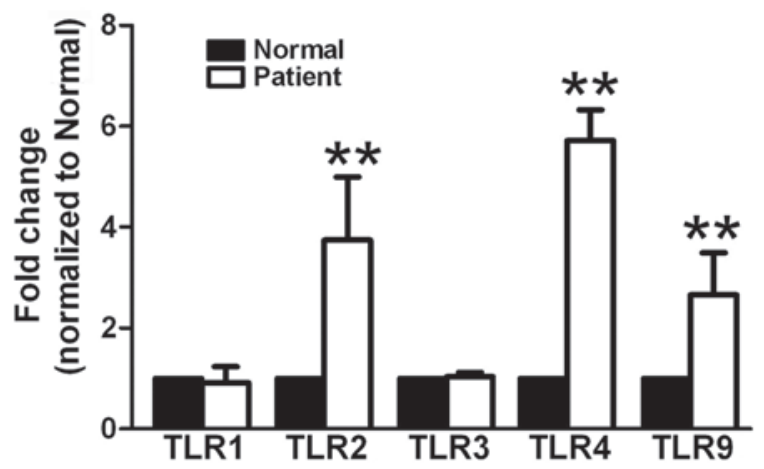

Figure 2. TLR mRNA expression in the mucosal biopsies of controls and patients with UC. ${ }^{* *} \mathrm{P}<0.05$, versus controls. TLR, Toll-like receptor.

plays a critical role as the front line of the mucosal innate immune system in the gastrointestinal tract (13-15).

The dysregulation of innate and adaptive intestinal immune responses to bacterial microbiota is believed to be the hallmark of IBD pathogenesis. In the present study it has been demonstrated that intestinal epithelial cells constitutively express several functional TLRs, which recognize a variety of distinct microbial components. These TLRs not only play a key role in microbial recognition in innate immunity but also participate in the activation of adaptive immune responses (16). Following the recognition of pathogen-associated molecular patterns by TLRs, signal transduction is initiated. This signaling pathway results in the activation of a number of transcription factors, including activator protein 1 , nuclear factor- $\kappa \mathrm{B}$, ETS domain-containing protein Elk-1, cyclic adenosine monophosphate-response element-binding protein and signal transducers and activators of tran- scription. As a result of the activation of the TLRs, major histocompatibility complex and costimulatory molecules are upregulated and proinflammatory cytokines and chemokines are expressed (17-19).

In the gastrointestinal tract, the polarized expression of TLRs in apical and basolateral compartments has been suggested to be a key factor underlying the discrimination between pathogenic bacteria invading the epithelium and nonpathogenic microbes facing the luminal surface (20-22). TLR ligation on the epithelial cells of the intestine by bacterial products induces epithelial cell proliferation, the secretion of immunoglobulin A into the gut and the expression of antimicrobial peptides (23).

In the present study, it was found that the gene expression of TLR 2 and TLR4 was significantly upregulated at the mRNA and protein levels in the inflamed mucosa as compared with the normal controls. By contrast, TLR1 and TLR3 levels were not found to be significantly altered in the patients with UC. These results corroborate those previously reported for TLR2 and TLR4 gene expression in IBD (5,6,17-19). Using immunofluorescence histochemistry, Cario and Podolsky (17) found that TLR3 expression was significantly downregulated in intestinal epithelial cells in active CD but not in UC. By contrast, significant upregulation of TLR4 was found in both UC and CD (17). In a study by Hausmann et al (18), the induction of TLR2, TLR4 and TLR5 mRNA was observed in inflammation-stimulated macrophages in the colonic mucosa of patients with IBD.

In the present study it was additionally found that TLR9 gene expression was upregulated in the mucosa of patients with UC as compared with the healthy controls, although in a previous study it was reported that TLR9 expression was downregulated in inflamed colonic mucosa from patients with 
IBD (24). In normal circumstances, TLR9 combines with unmethylated $\mathrm{CpG}$ motifs of the pathogen during bacterial or viral infection, thus stimulating the maturation and activation of dendritic cells and B cells and the elimination of pathogens by the secretion of cytokines and specific antibodies (25). In a study by Hall et al (26) it was reported that, in response to commensal bacterial DNA, dendritic cells signaled via TLR9 to inhibit the differentiation of regulatory $T$ cells in the gut. The activation of TLR9 has been shown to result in the maturation of dendritic cells and the release of type 1 T-helper cell cytokines, such as interleukin (IL)-6, IL-12, IL-10 and tumor necrosis factor- $\alpha$ (27-29). Consistent with the present results, previous studies have analyzed TLR9 expression in cells $(30,31)$, and found little TLR9 protein expression in isolated colonic epithelial cells, as assessed by western blot analysis. Gut inflammation with leukocyte infiltration may therefore play a role in the upregulation of TLR9 expression in the colonic mucosa of patients with UC.

In conclusion, the results of the present study have shown overexpression of TLR2, TLR4 and TLR9 in the colonic mucosa of patients with UC, which may be important in the biological pathogenesis of UC. The purpose of this receptor upregulation may be to increase the antigenic stimulation of inflammatory and immune pathways activated by ligand binding. Further studies are necessary to provide an enhanced understanding of the function of these TLRs in the mucosa from patients with UC.

\section{Acknowledgements}

This study was supported by the Natural Science Foundation of Heilongjiang Province (no. D201178).

\section{References}

1. Loftus EV Jr: Clinical epidemiology of inflammatory bowel disease: Incidence, prevalence, and environmental influences. Gastroenterology 126: 1504-1517, 2004.

2. Xavier RJ and Podolsky DK: Unravelling the pathogenesis of inflammatory bowel disease. Nature 448: 427-434, 2007.

3. Abreu MT: Toll-like receptor signalling in the intestinal epithelium: how bacterial recognition shapes intestinal function. Nat Rev Immunol 10: 131-144, 2010.

4. Akira S and Takeda K: Toll-like receptor signalling. Nat Rev Immunol 4: 499-511, 2004.

5. Szebeni B, Veres G, Dezsofi A, Rusai K, Vannay A, Bokodi G, Vásárhelyi B, Korponay-Szabó IR, Tulassay T and Arató A: Increased mucosal expression of Toll-like receptor (TLR)2 and TLR4 in coeliac disease. J Pediatr Gastroenterol Nutr 45: 187-193, 2007.

6. Frolova L, Drastich P, Rossmann P, Klimesova K and Tlaskalova-Hogenova H: Expression of Toll-like receptor 2 (TLR2), TLR4, and CD14 in biopsy samples of patients with inflammatory bowel diseases: upregulated expression of TLR2 in terminal ileum of patients with ulcerative colitis. J Histochem Cytochem 56: 267-274, 2008.

7. Chen LW, Chang WJ, Chen PH, Liu WC and Hsu CM: TLR ligand decreases mesenteric ishcemia and reperfusion injury-induced gut damage through TNF-alpha signaling. Shock 30: 563-570, 2008.

8. Berkowitz D, Peri R, Lavy A and Kessel A: Increased Toll-like receptor 9 expression by $\mathrm{B}$ cells from inflammatory bowel disease patients. Hum Immunol 74: 1519-1523, 2013.

9. Erridge C, Duncan SH, Bereswill S and Heimesaat MM: The induction of colitis and ileitis in mice is associated with marked increases in intestinal concentrations of stimulants of TLRs 2 , 4, and 5. PLoS One 5: e9125, 2010.
10. de Paiva NM, Ayrizono ML, Milanski M, Coope A, Oliveira LM, Fagundes JJ, Velloso LA, Coy CS and Leal RF: Differential expression of TLR2, TLR4 and JNK in mucosa of ileal pouches for ulcerative colitis. Is there a role for bacterial antigen pathway in asymptomatic patients? Int J Clin Exp Med 4: 179-186, 2011.

11. Livak KJ and Schmittgen TD: Analysis of relative gene expression data using real-time quantitative PCR and the 2(-Delta Delta C(T)) Method. Methods 25: 402-408, 2001.

12. Geremia A, Biancheri P, Allan P, Corazza GR and Sabatino A: Innate and adaptive immunity in inflammatory bowel disease. Autoimmun Rev 13: 3-10, 2014.

13. Mayer L, Eisenhardt D, Salomon P, Bauer W, Plous R and Piccinini L: Expression of class II molecules on intestinal epithelial cells in humans. Gastroenterology 100: 3-12, 1991.

14. Gomez CR, Boehmer ED and Kovacs EJ: The aging innate immune system. Curr Opin Immunol 17: 457-462, 2005.

15. Lavelle EC, Murphy C, O'Neill LA and Creagh EM: The role of TLRs, NLRs, and RLRs in mucosal innate immunity and homeostasis. Mucosal Immunol 3: 17-28, 2010.

16. Cario E, Rosenberg IM, Brandwein SL, Beck PL, Reinecker HC and Podolsky DK: Lipopoly-saccharide activates distinct signaling pathways in intestinal epithelial cell lines expressing Toll-like receptors. J Immunol 164: 966-972, 2000.

17. Cario E and Podolsky DK: Differential alteration in intestinal epithelial cell expression of toll-like receptor 3 (TLR3) and TLR4 in inflammatory bowel disease. Infect Immun 68: 7010-7017, 2000.

18. Hausmann M, Kiessling S, Mestermann S, Webb G, Spöttl T, Andus T, Schölmerich J, Herfarth H, Ray K, Falk W and Rogler G: Toll-like receptors 2 and 4 are up-regulated during intestinal inflammation. Gastroenterology 122: 1987-2000, 2002.

19. Toiyama Y, Araki T, Yoshiyama S, Hiro J, Miki C and Kusunoki M: The expression patterns of Toll-like receptors in the ileal pouch mucosa of postoperative ulcerative colitis patients. Surg Today 36: 287-290, 2006.

20. Gewirtz AT, Navas TA, Lyons S, Godowski PJ and Madara JL: Cutting edge: bacterial flagellin activates basolaterally expressed TLR5 to induce epithelial proinflammatory gene expression. J Immunol 167: 1882-1885, 2001.

21. Gewirtz AT, Simon PO Jr, Schmitt CK, Taylor LJ, Hagedorn CH, O'Brien AD, Neish AS and Madara JL: Salmonella typhimurium translocates flagellin across intestinal epithelia, inducing a proinflammatory response. J Clin Invest 107: 99-109, 2001.

22. Xavier RJ and Podolsky DK: Microbiology. How to get along - friendly microbes in a hostile world. Science 289 : 1483-1484, 2000.

23. Shaykhiev R, Behr J and Bals R: Microbial patterns signaling via Toll-like receptors 2 and 5 contribute to epithelial repair, growth and survival. PLoS One 3: e1393, 2008.

24. Pedersen G, Andresen L, Matthiessen MW, Rask-Madsen J and Brynskov J: Expression of Toll-like receptor 9 and response to bacterial $\mathrm{CpG}$ oligodeoxynucleotides in human intestinal epithelium. Clin Exp Immunol 141: 298-306, 2005.

25. Asselin-Paturel C and Trinchieri G: Production of type I interferons: plasmacytoid dendritic cells and beyond. J Exp Med 202: 461-465, 2005.

26. Hall JA, Bouladoux N, Sun CM, Wohlfert EA, Blank RB, Zhu Q, Grigg ME, Berzofsky JA and Belkaid Y: Commensal DNA limits regulatory $\mathrm{T}$ cell conversion and is a natural adjuvant of intestinal immune responses. Immunity 29: 637-649, 2008.

27. Lee J, Rachmilewitz D and Raz E: Homeostatic effects of TLR9 signaling in experimental colitis. Ann NY Acad Sci 1072: 351-355, 2006.

28. Ewaschuk JB, Backer JL, Churchill TA, Obermeier F, Krause DO and Madsen KL: Surface expression of Toll-like receptor 9 is upregulated on intestinal epithelial cells in response to pathogenic bacterial DNA. Infect Immun 75: 2572-2579, 2007.

29. Ghadimi D, Vrese M, Heller KJ and Schrezenmeir J: Effect of natural commensal-origin DNA on toll-like receptor 9 (TLR9) signaling cascade, chemokine IL-8 expression, and barrier integritiy of polarized intestinal epithelial cells. Inflamm Bowel Dis 16: 410-427, 2010.

30. Lee J, Mo JH, Katakura K, Alkalay I, Rucker AN, Liu YT, Lee HK, Shen C, Cojocaru G, Shenouda S, Kagnoff M, Eckmann L, Ben-Neriah Y and Raz E: Maintenance of colonic homeostasis by distinctive apical TLR9 signalling in intestinal epithelial cells. Nat Cell Biol 8: 1327-1336, 2006.

31. Akhtar M, Watson JL, Nazli A and McKay DM: Bacterial DNA evokes epithelial IL-8 production by a MAPK-dependent, NF-kappaB-independent pathway. FASEB J 17: 1319-1321, 2003. 